\title{
IOT INTEGRATED SMART STREET FURNITURE: A CASE STUDY OF UNIVERSITY, INDUSTRY AND LOCAL GOVERNMENT COLLABORATION
}

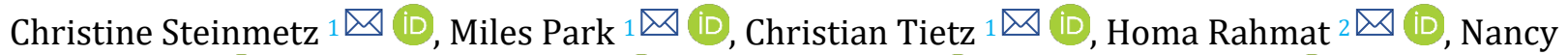

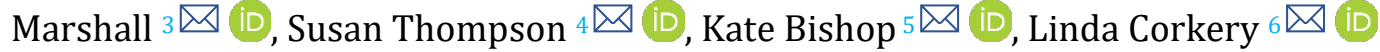

Senior Lecturer, School of Built Environment, University of New South Wales, Kensington, NSW, Australia

${ }^{2}$ Lecturer, School of Built Environment, University of New South Wales, Kensington, NSW, Australia

${ }^{3}$ Associate Professor, Sydney School of Architecture, Design and Planning, University of Sydney, Sydney,

NSW, Australia

${ }^{4,6}$ Professor, School of Built Environment, University of New South Wales, Kensington, NSW, Australia

Associate Professor, School of Built Environment, University of New South Wales, Kensington, NSW

Australia

\section{ABSTRACT}

Received 1 September 2021

Accepted 15 September 2021

Published 11 December 2021

\section{CorrespondingAuthor}

Christine Steinmetz,

c.steinmetz@unsw.edu.au

DOI 10.29121/IJOEST.v5.i6.2021.245

Funding: This research has been funded by the Australian Government, Department of Industry, Innovation and Science, Department of the Prime Minister \& Cabinet in the Smart Cities and Suburbs Program, 2017 and 2018.

Copyright: (C) 2021 The Author(s). This is an open access article distributed under the terms of the Creative Commons Attribution License, which permits unrestricted use, distribution, and reproduction in any medium, provided the original author and source are credited.

OPEN ACCESS
This article discusses the design process and pilot program of a suite of IoTintegrated street furniture aimed to improve use and amenity of municipality assets in public open spaces in Sydney, Australia. Networked sensors were embedded in the furniture and linked to a web-based dashboard application enabling a digital twin of the asset to monitor and analyze how and when the furniture was used. The prototype and modifications to existing furniture designs provided additional utility for the local community through lighting, free wi-fi access, power outlets, USB charging, water, a weather station and bench space. Outcomes of the street-furniture installation revealed innovative protocols for design-development teams and asset managers to review product performance and efficiency. This article presents a collaborative government/industry/university project that has been recognized by The World Bank for intelligent neighborhood design practices and by the Planning Institute of Australia for its novel approach to community social infrastructure.

Keywords: IoT, Street Furniture, Smart Products, Local Government, Public Space

\section{INTRODUCTION}

In 2019, The Economist (2019) predicted that by 2035 the world will have one trillion products and devices connected to computers. As technologies evolve at a rapid pace, including the ability to connect to anything (i.e., household products and public infrastructure) at anytime and anywhere, we look to an emerging technology of interconnected devices that communicate in a network, generally described as the Internet of Things (IoT) Li et al. (2015), Madakam et al. (2015). In 2014, the inaugural issue of IEEE Internet of Things Journal opened with a proposition from IEEE Life Fellow Professor John Stankovic, who said the IoT would become "a utility with increased sophistication in sensing, actuation, communications, control and in creating knowledge from vast amounts of data ... result[ing] in qualitatively different lifestyles from today" Stankovic (2014). Stankovic was correct, and we have reached the tipping point where the IoT, once limited to 
the technology industry, is reaching a mainstream audience of everyday users.

In the past six years, the marketplace has been flooded with products and electrical devices that contain embedded sensors and microprocessors interconnected through digital networks. This technology is part of the growing trend of creating inanimate objects that are "smart," providing opportunities for products and services to become more functional in design, more sustainable in materials and more adaptable to consumer needs, forming "totally new and unpredictable services" Stankovic (2014). These often low-cost and low-power sensor and microprocessor devices can be applied to a range of products, in public, private and commercial contexts, for a multitude of purposes and within almost any type of landscape-from the home and urban public open spaces to rural and wilderness areas. In an urban setting, IoT provides a "unified, simple, and economical access to a plethora of public services, thus unleashing potential synergies and increasing transparency" offering "services to citizens, companies, and public administrations" Zanella et al. (2014). However, the accessibility and affordability of IoT applications allows even small government municipalities to benefit from smart technologies offering citizens the ability to engage with and be connected to their community through hard and soft digital infrastructure.

For product developers, IoT not only offers opportunities to discover new ways to integrate products but can also assist with decision-making in the design process by using prototypes and models embedded with IoT sensors that provide real-time feedback. Although much of this development work resides within specialist communities and disciplines such as computer science, software engineering and data analytics, with the advent of low-cost open-source IoT sensors, networks and platforms the field is attracting designers and creators from non-specialist fields. These sensor-equipped products, sensor networks and platforms can also offer effective, financially savvy and timesaving systems to owners and asset managers of public infrastructure such a facilities management department in local government.

This article critically evaluates Smart Social Spaces, a collaborative research project involving a local government authority, a street-furniture manufacturer and a team of university researchers, all located in the Sydney metropolitan area. This government/industry/university team created new, and modified existing, street furniture using IoT technologies. The UNSW design-led project was a novel collaborative initiative with the local council and has been internationally recognized by the World Bank and others as a model of best practice for incorporating technology in urban public open spaces Kaw et al. (2020), Cherney (2018).

\section{IoT for Design}

Incorporating IoT into product design is a sophisticated research tool for gathering information about product performance and use before committing to the costs of a finalized design and sending it into the marketplace. In a design scenario in which there is a tangible output, IoT technology can improve what is traditionally an iterative process and user-centered approach of testing and product interaction with prototypes and mock-ups. As mentioned by Tao et al. (2019), incorporating data collected from IoT integrated products into the design process is a new paradigm for makers and designers. Similarly, Gianni, Mora and Divitini Gianni et al. (2019) argue that IoT applications in the design process can be collaborative in that makers and designers, as non-expert developers, "can be part of a participatory design-orientated strategy" to reveal new opportunities in IoT technologies. 
Few studies have investigated a digital twin for product design in which the communication and interaction between a physical product and its digital representation enable a more informed design process Tao et al. (2019). Datadriven design gathers sensor data on a product's performance and behavior and its interaction with users. In a new concept of the digital twin, the physical product is linked or paired to a virtual product and characterized by the two-way interactions between the digital and physical worlds, linking virtual visualization with physical metrics derived from IoT sensors. The availability of low-cost IoT technologies and the maturity of augmented reality (AR) and virtual reality (VR) technologies enable the concept to be applied to an unlimited number of products in a variety of contexts. This has multiple benefits: for product-development teams in simulating physical functions before they are built; for asset managers in detecting if any repairs, maintenance or changes are needed to the physical version; and for users who may access product features through their phones or tablets.

\section{Methods: Smart City Case Study: Integrated Street Furniture}

Smart-city products and services applied in urban settings have become synonymous with IoT Theodoridis et al. (2013), Al-Fuqaha et al. (2015). City planners, architects, engineers, policymakers, and decision-makers are invested in the concept of smart cities and the application of IoT technologies to improve services and liveability and to track services such as building structural health, air quality, waste management, noise monitoring, traffic management, energy consumption, parking, and lighting. As a tool, IoT can measure and lower expenditures through analysis and reporting of site or system changes that offer potential savings Zanella et al. (2014). Most importantly, these technologies can contribute to providing real-time and historic series data to support evidence-based decision-making. In addition to planning the smart city, asset management is also seen as a sector that can benefit from smart products and IoT technologies Zanella et al. (2014), Difallah et al. (2013).

The Smart Social Spaces Kaw et al. (2020) project was realized over three years through two Australian Commonwealth government-awarded Smart Cities and Suburbs Program grants (2017 and 2018) awarded to the University of New South Wales Sydney, Georges River Council, and Street Furniture Australia. The government/industry/university partnership provided an opportunity to design, test and implement new smart street furniture integrated with IoT sensors in the public domain and to pilot a dashboard to manage these municipality assets. Aims of the collaboration were to produce and test smart street furniture that would, firstly, support social health and, more specifically, improve the amenity and use of public open spaces in Georges River Council local government area; improve the asset management system for the Council facilities department; form a meaningful collaboration of academics, industry practitioners and local government officials; provide an evidence-base for the design-development process and design; and develop new smart street furniture that can be placed in situ.

The team was guided by smart-city best-practice case studies Batty et al. (2012), Toch and Feder (2016), van et al. (2016), Trowbridge (2019), Steinmetz and Oren (2020) observation and behavior mapping studies at the two case-study sites Bishop et al. (2019a), Bishop et al. (2019b) and the underlying premise of the grant: the desire to promote healthy and connected living through innovative streetfurniture designs. Working as a multidisciplinary team, and engaging with designers, facility managers, software developers and university researchers, the team developed a suite of products with Street Furniture Australia by incorporating sensors and networking technologies into an existing prototype of a new street 
furniture product line. The IoT sensor-equipped street furniture-rubbish bins, seating, picnic tables, barbeques, lighting and water taps (see Figure 1)-were placed in two highly visible and active local community areas to study the impact on use of the public open-space environments, to provide data to help improve provision and social amenity, and to provide feedback to the asset management system-the dashboard.

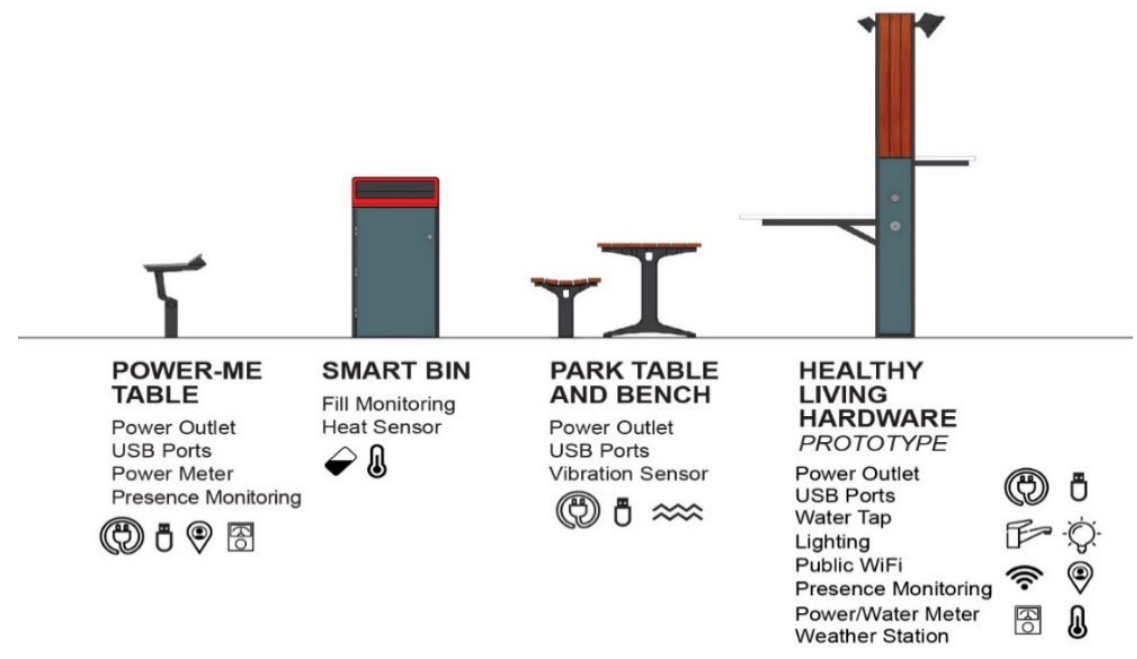

Figure 1 Suite of Street Furniture

Materials: Heathy Living Hardware (HLH) and Street Furniture Assets

A key addition to the suite of street furniture was the development and testing of a human-scale street pole called Healthy Living Hardware (HLH) (see Figure 2). Ten years ago, a variation of the HLH (then called the Yardmaster) was a design response to chronic overcrowding and ill-performing houses in remote Indigenous communities in Australia Tietz (2012). The Yardmaster furniture acted as infrastructure to assist daily activities that would typically occur in houses and spill out into the open yards, providing water, power, lighting and food-preparation surfaces. A modified version, the HLH, is equipped with sensors to monitor power, lighting and water usage, and the presence of people (detected via mobile phone MAC address) in the immediate vicinity of the HLH. The sensors on each piece of furniture are connected via multiple wireless networks to cloud servers and to the app-based Smart Asset Management (SAM) dashboard created during the scope of the project. 


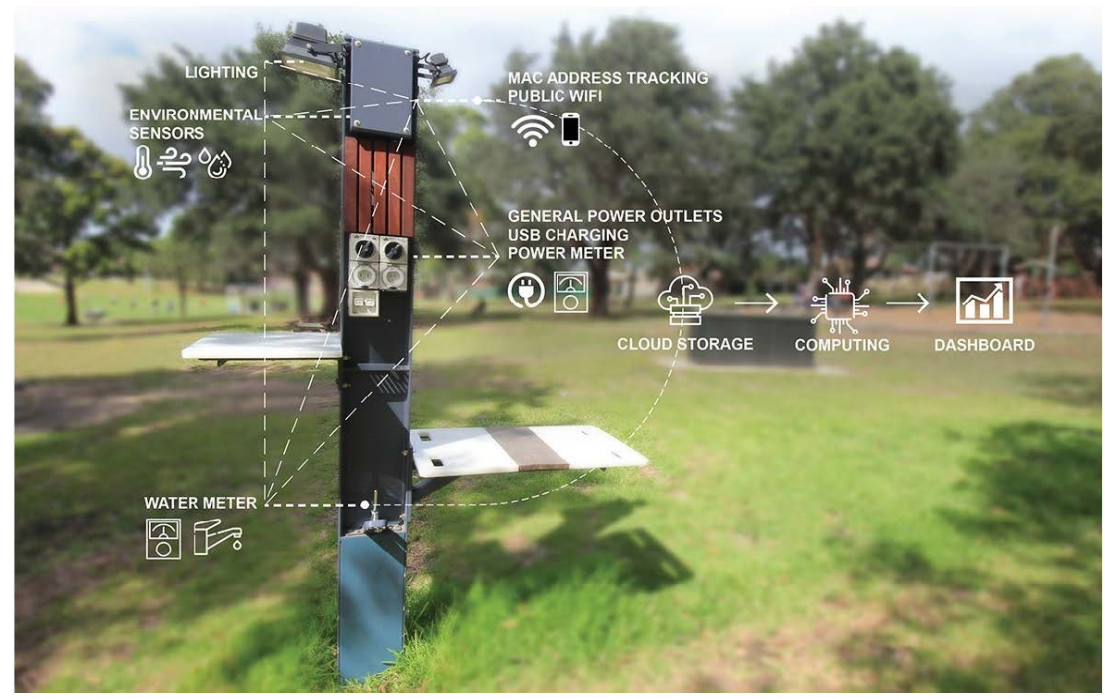

Figure 2 HLH Pole Physical and Digital Assets

The HLH's function is to improve amenity in public spaces, providing lighting, free wi-fi access, power outlets, USB charging, water, a weather station and bench space (Figure 1). It provides data on product use and interaction: recording power and water use and micro-climatic conditions and counting smart phone devices as a surrogate for people proximity and dwell time using MAC address tracking. The data collected from the sensors is uploaded to a cloud repository where it is processed, filtered and presented as a live feed on the web-based dashboard, which is accessible to the Council's asset managers and the researchers. The HLH pole was prototyped and tested at two locations in the Georges River Council area-a park and a civic square-and was combined with pre-existing and newly installed furniture (picnic table seating, bench seating, bins and barbeques).

Sensors were embedded in the street-furniture assets to measure a range of real-time-event and environmental information. Smart bins could detect bin fullness to facilitate efficient scheduling for emptying and bin internal temperature (in the case of fire). The HLH pole included sensors with MAC address tracking, sensors that monitored the water and electricity hook-up, the presence of people near seating areas, power consumption of electric barbeques and seating arrangements (detected if the seating was being used). Figure 3 shows sensor types linked to specific furniture assets. 


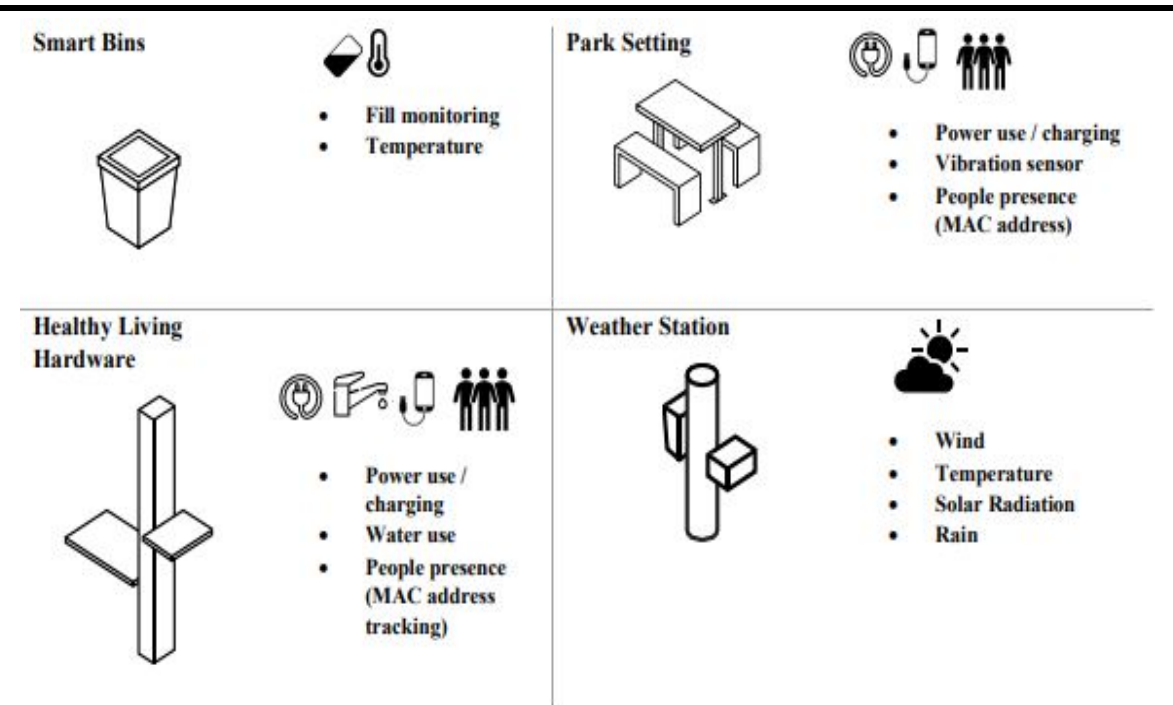

Figure 3 Furniture Assets and Sensor Allocation

\section{Network and the SAM Dashboard}

In most instances, sensor data was uploaded in real-time via wireless networks to specific cloud platforms. The collected data was then filtered and aggregated from the cloud platforms and presented on the custom-built SAM Dashboard, as shown in Figure 4. The use of multiple sensor types, networks and cloud platforms added significant complexity to the project but offered an opportunity to test the advantages and disadvantages of multiple data feeds using different devices and carrier platforms. For example, we tested a combination of LAN (local area network) devices connected to the wider network via SIGFOX as well as several parallel LTE/3G/4Gs.

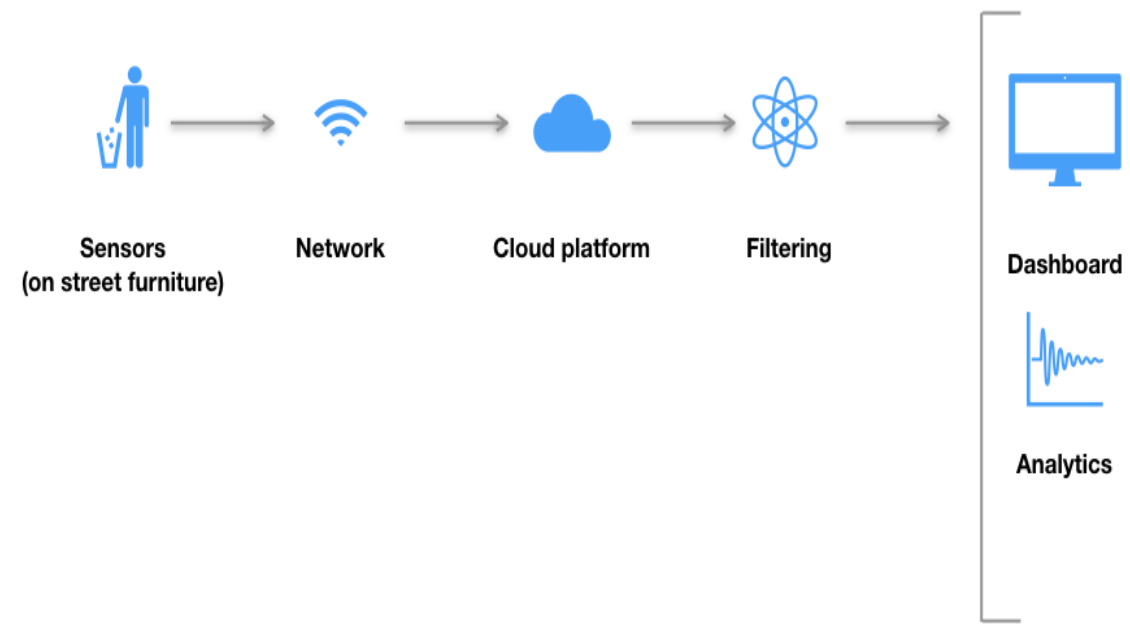

Figure 4 Smart Social Spaces Systems Overview from the Sensors to SAM Dashboard

The SAM Dashboard was developed to present filtered and aggregated data. The data for this dashboard were acquired via the cloud platforms associated with each sensor's vendor. Machine-to-machine translations of the raw data (vendor API and website scraping) were collated and filtered for display on the SAM Dashboard, which served as an information hub and management tool for different audiences. For the community and public, it offered microclimate information for specific 
locations where the HLH and street furniture were located. For the design team, manufacturer and researchers, it offered time-series data on a range of metrics captured by the sensors-visitations (via the proxy of MAC address registration) and water and power use. For asset managers, in this case the local government authority, it monitored physical assets and their use, management and maintenance through the digital twin.

\section{DISCUSSION}

\section{Design and Prototyping}

IoT-enabled prototypes offer design teams a means to gain high-fidelity feedback in real time during the design development of a new product or service. Product performance and environmental measurements via onboard networked sensors can be obtained from actual use in situ, enhancing the quality of feedback during the initial testing of a design or for the commissioning of a newly installed product. An advantage of this approach is that the sensors can provide an authentic reading of user interaction and feedback and are not clouded by simulation or lab settings using a prearranged group of users. Real-time data capture over a sustained period can provide continuous time-series monitoring without the need for a ream of observers.

This approach is common with developing software applications since stability and usability shortcomings can be rectified with successive software updates. The physical world of IoT is not so fluid, but this approach of automated sensing of product use offers a valuable step in the product-design verification process. It supports evidence-based decision-making in the design process. It can also enrich the quality of learning from prototyped designs as analysis of collected data can pinpoint deficiencies in a design. For manufacturers of street furniture, IoT provides new business opportunities to expand product-management services and product sales. For product-development teams, sensors onboard a prototype feed data to a remote web-based dashboard and offer real-time information to expose deficiencies and provide insights about a prototype's performance. Prototypes equipped with sensors indicate points of human interaction with the product, localized environmental conditions, and product performance and reliability characteristics.

If IoT is to be an integral part of a product-design solution, as with the HLH and other street furniture assets discussed in this paper, all sensors and data feeds need to be fully tested and operational before installation. This will not only ensure that the product will perform as designed but will also test and help validate project partners' and IoT vendors' abilities and expertise in this emergent field. IoT technologies are novel territory for most designers and require new skills and knowledge. In addition to existing design documentation practices (production drawings, product specifications, etc.), updated forms of technical documentation are also required for IoT projects. This includes developing systems diagrams of the IoT stack and technical information of the sensors and digital platforms.

\section{Managing IoT Sensors and Data}

Technology does not come without its challenges. As IoT applications proliferate, it is important to recognize and identify the risks and negative impacts this technology may bring or amplify, notably, growing concerns about surveillance and the loss of privacy Oleshchuk (2009), Peppet (2014) and the potential for misuse by governments and corporations in addition to security issues Khan and Salah (2018), Xu et al. (2014). In ideal circumstances, the selection of an IoT platform would provide data feeds for what needs be measured, managed or 
monitored. However, due to practical, ethical, legal and privacy reasons, fully integrated "off the shelf" solutions were not readily available for the combination of desired metrics we sought to learn from the street furniture.

Cost, system complexity and the lack of standards for interoperability also needed to be considered in this project. Using multiple IoT platforms supplied by a variety of providers was both a strength and weakness of the project. While a mixed IoT system enabled the project team to evaluate performance and usefulness of different sensors and IoT platforms, it did introduce significant and unnecessary complexity. A weakness of this approach was that it required managing multiple systems in parallel, utilizing differing protocols, networks, data formats and sampling frequency. An ongoing challenge encountered during the project was maintaining operational sensors and providing a stream of useful data. The stability of any IoT system is only as good as the weakest link in the chain, and power outages, wi-fi dropouts and vandalism were detected faults that caused loss of data. In some cases, data feeds dropped out for no detectable reason and then come back online.

Merely collecting data from sensors is not enough-the output must be interpretable and useful and continually re-evaluated. The quality of data (not just the quantity) was critical so as not to overwhelm the data management of the project. Data noise needed to be filtered, and data feeds from sensors needed to be normalized to be presented on the SAM Dashboard. Some of the specified sensors for the project were provided by vendors that operated within a closed IoT platform that linked the elements of the IoT stack, managing and presenting the data stream from sensors to dashboard. The advantage of this approach was the increased likelihood of a robust and reliable IoT solution. But loss of data interoperability and ongoing service or licensing costs can be substantial when multiple sensors are used over a long period. Sensors and open IoT platforms offered the most flexibility as data could be harvested via an API or scrapped from the vendors server or web application to be exported to our developed SAM Dashboard.

Interoperability and standardization pose substantial challenges for the IoT industry, which remains fragmented in a field of competitive platforms with many providers, akin to the early days of computing with competing incompatible operating systems. The reliance on proprietary (pre IoT) wireless platforms (3G/4G LTE) initially slowed the adoption of IoT systems. But with newer IoT-dedicated networks (NB-IoT, LTE CatM1, LoRaWAN and SIGFOX) becoming available, IoT deployments will become lower in cost, require less power and be available with a greater geographical coverage.

An important element of the project was to understand when (de-identified) people were using or interacting with the street furniture. This was partially achieved by using vibration sensors in park seating arrangements, however, detecting the presence of people using or interacting with other furniture assets presented a challenge. With the growth in community concern over surveillance and the requirements of the research project to adhere to ethical, privacy and legal policies, a compliant method needed to be adopted for this project that avoided identifying or profiling individuals or conducting any related activity other than just counting people. We used MAC address tracking to log the presence of a digital device that a person might be carrying in the vicinity of the furniture. Each digital device had a unique (MAC) address used for communicating with networks and other devices. We used this as an indicator of the number of people in the close vicinity of the HLH. However, it was only an approximate indicator as individuals could be carrying multiple devices or be carrying none. A practical problem we encountered with this method was that the initial batch of data we received indicated very high readings, around 60,000 users. On investigation, we discovered 
that the radius sensitivity range for the MAC scanning was capturing passengers from an adjacent train station.

\section{Managing IoT Projects}

Designing and managing smart-city projects requires interdisciplinary solutions. Many specializations have a vital role to play in delivering successful project outcomes. The Smart Social Spaces project involved researchers, planning practitioners, industrial designers, technologists, data scientists, landscape architects, social scientists, project planners and council tradespeople. Roles and responsibilities for the installation, commissioning and maintenance were established in advance, but a challenge for this new IoT project was that some required roles did not exist or they fell between existing specialist roles.

Our council-managed IoT street-furniture asset was designed to be the remit of public works or park maintenance teams, with the sensors and data management being handled by the IT department. A digital twin of physical assets necessitated new cross-disciplinary skills and responsibilities for municipality departments. IoT introduces a new layer of digital infrastructure that requires significant extra resources, investing in new technology integration and building new expertise to manage technology assets. In current circumstances, IoT integration remains as a custom-built service for any given project, a result of its cost and complexity.

Expertise and training to manage and deliver IoT projects needs to be developed. For designers and design educators, this presents opportunities for new skills and knowledge in designing IoT products, such as designing and documenting embedded sensors, developing digital twins, and data presentation. For asset managers, it requires developing new expertise for maintaining and managing IoTequipped products. For ongoing successful service delivery, what may appear to be simple housekeeping, such as the management of device passwords, API codes, SIM card numbers and service provider expiration dates, needs to be documented and managed, from the design research team's handover to the implementation by the operations and maintenance owner.

\section{CONCLUSION}

The primary aim of the case study presented here was to improve the amenity and use of public open spaces for the community of Georges River Council. Often, IoT projects are framed almost exclusively through technological parameters. While new IoT technologies feature heavily in this discussion, they exist to enhance a product or service experience by putting people first. We have learnt that the delivery of IoT smart products requires interdisciplinary teams to negotiate complex sociological, technological, ethical and managerial dimensions. For product designers, embedding IoT sensors in prototype designs offers a new tool to evaluate an evolving design, but this requires new skills and knowledge. Additionally, sensorequipped smart products combine a digital representation with a physical product. Real-time simultaneous management via a web-based dashboard of a physical asset opens many new possibilities for interaction and management of a product or service as a system.

This project offers a workable model of how smart street furniture represents the micro infrastructure of the smart city. Key findings from this project include the novelty of using sensors on public asset infrastructure, such as street furniture, to digitally track public space usage-not only of the asset but also the broader amenity that it provides. The project findings also acknowledge the importance of testing all systems before installation, avoiding complexity where possible, 
collecting quality data (over quantity) of what needs to be measured, enhancing documentation for handover and management, and developing new assetmanagement systems.

This work was funded by the Australian Government, Department of Industry, Innovation and Science, Department of the Prime Minister \& Cabinet in the Smart Cities and Suburbs Program under grant number SCS59323 (2017 and 2018).

\section{CONFLICT OF INTEREST AND ACKNOWLEDGEMENTS}

1) The authors do not have any conflict of interest with parties engaged in this work.

2) The authors would like to recognise Street Furniture Australia as our industry partner and Georges River Council, as our government partner.

3) No ethics application was required in this research.

\section{REFERENCES}

Al-Fuqaha, A., Guizani, M., Mohammadi, M., Aledhari, M., \& Ayyash, M. (2015). Internet of things: A survey on enabling technologies, protocols, and applications. IEEE Communications Surveys and Tutorials, 17(4), 23472376. Retrieved from https://doi.org/10.1109/COMST.2015.2444095

Batty, M., Axhausen, K. W., Giannotti, F., Pozdnoukhov, A., Bazzani, A., Wachowicz, M., Ouzounis, G., \& Portugali, Y. (2012). Smart cities of the future. European Physical Journal Special Topics, 214(1), 481-518. Retrieved from https://doi.org/10.1140/epjst/e2012-01703-3

Bishop, K., Rahmat, H., Marshall, N., Corkery, L., C., Park, M., Steinmetz, M., Tietz, C., Thompson, S. (2019a). Behaviour mapping report 2: Smart social spaces: Smart street furniture supporting social health. University of New South Wales Sydney.

Bishop, K., Rahmat, H., Marshall, N., Corkery, L., Park, M., Steinmetz, C., Tietz, C., \& Thompson, S. (2019b). Behaviour mapping report: Smart social spaces: Smart street furniture supporting social health. University of New South Wales Sydney.

Cherney, M. (2018, June 26). There are park benches. And then there are smart park benches. Wall Street Journal. Retrieved from https://www.wsj.com/articles/there-are-park-benches-and-then-thereare-smart-park-benches-1530064801

Difallah, D. E., Cudre-Mauroux, P., \& McKenna, S. A. (2013). Scalable anomaly detection for smart city infrastructure networks. IEEE Internet Computing, 17(6), 39-47. Retrieved from https://doi.org/10.1109/MIC.2013.84

Gianni, F., Mora, S., \& Divitini, M. (2019). RapIoT toolkit: Rapid prototyping of collaborative Internet of Things applications. Future Generation Computer Systems, 95, 867-879. Retrieved from https://doi.org/10.1016/j.future.2018.02.030

Kaw, J. K., Anderson, C., \& Soukup, T. (2020). Evidence-based planning and design. In J. K. Kaw, H. Lee \& S. Wahba (Eds.), The hidden wealth of cities: Creating, financing, and managing public spaces (Chapter 4, pp. 97-108). World Bank. Retrieved from https://doi.org/10.1596/978-1-4648-1449-5_ch4

Khan, M. A., \& Salah, K. (2018). IoT security: Review, blockchain solutions, and open challenges. Future Generation Computer Systems, 82, 395-411. Retrieved from https://doi.org/10.1016/j.future.2017.11.022 
Li, S., Da Xu, L. D., \& Zhao, S. (2015). The internet of things: A survey. Information Systems Frontiers, 17(2), 243-259. Retrieved from https://doi.org/10.1007/s10796-014-9492-7

Madakam, S., Ramaswamy, R., \& Tripathi, S. (2015). Internet of Things (IoT): A literature review. Journal of Computer and Communications, 03(5), 164173. Retrieved from https://doi.org/10.4236/jcc.2015.35021

Oleshchuk, V. (2009). Internet of things and privacy preserving technologies. In 1st International Conference on Wireless Communication, Vehicular Technology, Information Theory and Aerospace and Electronic Systems Technology (WirelessVITAE'09) (pp. 336-340). Piscataway, New Jersey, IEEE. $\quad$ Retrieved from https://doi.org/10.1109/WIRELESSVITAE.2009.5172470

Peppet, S. R. (2014). Regulating the internet of things: First steps toward managing discrimination, privacy, security and consent. Texas Law Review, 93, 85176.

Stankovic, J. A. (2014). Research directions for the internet of things. IEEE Internet of Things Journal, 1(1), 3-9. Retrieved from https://doi.org/10.1109/JIOT.2014.2312291

Steinmetz, C., \& Oren, H. (2020). Tel Aviv: Making place through technology. In K. Bishop \& N. Marshall (Eds.), The Routledge Handbook of People and place in the 21st century city (pp. 219-228). Routledge. Retrieved from https://doi.org/10.4324/9781351211543-24

Tao, F., Sui, F., Liu, A., Qi, Q., Zhang, M., Song, B., Guo, Z., Lu, S. C. -Y., \& Nee, A. Y. C. (2019). Digital twin-driven product design framework. International Journal of Production Research, 57(12), 3935-3953. Retrieved from https://doi.org/10.1080/00207543.2018.1443229

The Economist. (2019, September 14). Chips with everything: How the world will change as computers spread into everyday objects. Retrieved from https://www.economist.com/leaders/2019/09/12/how-the-world-willchange-as-computers-spread-into-everyday-objects

Theodoridis, E., Mylonas, G., \& Chatzigiannakis, I. (2013). Developing an IoT smart city framework. Information, Intelligence, Systems and Applications (IISA), 2013, 1-6. Retrieved from https://doi.org/10.1109/IISA.2013.6623710

Tietz, C. (2012). The yardmaster. Industrial Design Educators Network, 1, 50-52.

Toch, E., \& Feder, E. (2016). International case studies of smart cities: Tel Aviv, Israel. Discussion Paper no. IDB-DP-444. Institutions for Development Sector, Fiscal and Municipal Management Division: Inter-American Development Bank. Retrieved from https://doi.org/10.18235/0000416

Trowbridge, L. (Ed.). (2019). A better world: Actions and commitments to the sustainable development goals (vol. 5). Tudor Rose.

Van Waart, P., Mulder, I., \& de Bont, C. (2016). A participatory approach for envisioning a smart city. Social Science Computer Review, 34(6), 708-723. Retrieved from https://doi.org/10.1177/0894439315611099

$\mathrm{Xu}, \mathrm{T} ., \mathrm{Wendt,}$ J. B., \& Potkonjak, M. (2014). Security of IoT systems: Design challenges and opportunities. Proceedings of the 2014 IEEE/ACM International Conference on Computer-Aided Design (pp. 417-423). IEEE Publications. Retrieved https://doi.org/10.1109/ICCAD.2014.7001385

Zanella, A., Bui, N., Castellani, A., Vangelista, L., \& Zorzi, M. (2014). Internet of things for smart cities. IEEE Internet of Things Journal, 1(1), 22-32. Retrieved from https://doi.org/10.1109/JIOT.2014.2306328 
